\title{
Optical path pointing error and coaxiality analysis of APT system of space laser communication terminal
}

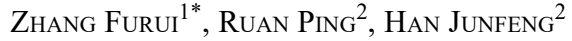 \\ ${ }^{1}$ College of Optoelectronic Engineering, Xi'an Technological University, \\ Xi'an 710021 , China \\ ${ }^{2} \mathrm{Xi}$ 'an Institute of Optics and Precision Mechanics (XIOPM), \\ Chinese Academy of Sciences (CAS), \\ Xi'an 710119, China \\ ${ }^{*}$ Corresponding author: jiffring@126.com
}

\begin{abstract}
Precision beam pointing is the key indicator for APT (acquisition, pointing and tracking) system in space laser communication. The laser travels inside the optical system and the pointing vector will be affected by an assembly error of the axis and reflectors. In this paper, the model of the optical path pointing error and coaxiality error induced by the assembly error are established; the error distribution is given and a quantitative analysis is performed. The results show that the magnitude of pointing error is affected by the axis assembling error greatly but its distribution is susceptible to the reflector assembly error. Finally, the correction of coaxiality is performed and tested. The experimental results show that the coaxiality error can be greatly improved and the mean value of the coaxiality error of a beacon path and a signal path are 14 and $9.6 \mu \mathrm{rad}$, respectively, which meets the requirements. This work can provide guidance for design and assembly of the APT and contribute to the improvement of its pointing performance.
\end{abstract}

Keywords: pointing error, coaxiality error, APT system, space laser communication.

\section{Introduction}

In the last decades, there has been an unprecedented interest in the field of satellite laser communication, which became one of the most attractive areas of communication. The satellite laser communication technology is expected to conquer the bottleneck of traditional microwave communication for the advantages of higher date rate, longer distance communication, smaller size, lighter weight, lower cost, etc. [1-4]]. However, due to the beam width which is very narrow and the link distance which is so long, it requires highly pointing accuracy to ensure stable communication during duplex communication. Therefore, it needs special system which is called APT (acquisition, pointing and tracking) to control the outgoing beam (OB) aimed at the counter terminal and to stabilize the incoming beam (IB) to stay on detectors. 
In a typical APT system, five optical paths are required to realize acquisition, pointing, tracking and communication functions which are beacon transmit path, coarse tracking path, signal transmit path, signal receive path and fine tracking path. Different optical paths have different functions. The pointing precision of beacon beam will affect acquisition probability, and the pointing precision of a signal beam will affect the stability of the point ahead link and determine whether the communication link will be established successfully. Therefore, highly beam pointing precision is the prerequisite for duplex communication [ $[$ ].

In the past, most research papers paid attention to atmospheric turbulence induced pointing error $(\mathrm{PE})$ [6] , detector angular error []], wavefront error induced by structural deformation []. But the influence of the assembling error on PE has not been analyzed in detail. The PE are most influenced by assembly precision of the axis and reflectors in APT. The axis error will deflect the beam axis from the machinery of CPA (coarse pointing assembly) and then PE will change along with the axis rotate angle (azimuth $\theta_{\mathrm{az}}$ and elevation $\theta_{\mathrm{el}}$ ). The reflectors' assembly error will generate static accumulative PE. Therefore, it is necessary to study the relationship between the assembly error and PE. Additionally, the angle between $\mathrm{OB}$ and IB is one of the key indicator that represents coaxiality. In paper [9], the pointing error induced by the axis error is analyzed and the correction is performed, but the reflector errors are not taken into consideration and the coaxiality is not mentioned. CHEN et al. studied the influence of polarization characteristics of a cube corner reflector (CCR) on coaxiality detection [ $[$ ], but he did not take assembly error into consideration. QIAN tested the coaxiality of the designed coaxial system, but the assembly errors are not mentioned yet [12]. In this paper, we established the PE analysis model of the APT system based on the vector optical method, and the influence regular is analyzed and discussed. For the first time, we analyzed the influence of the assembly error on coaxiality, derived out the model, analyzed the influence relation and performed the experiments. Based on the study, we can analyze the PE and coaxiality quantitatively and give some guidance for the APT system design and assembling.

This paper has the following outline. In Section 2 the installing error has been analyzed. Section 3 builds the PE of beacon laser and signal laser, based on this the coaxiality is analyzed. The assembling and correction process is performed in Section 4 . The conclusion is given in Section 6 .

\section{APT system description}

For convenience of reading, most acronyms used in this paper are listed in the Table.

The internal optical path structure of a typical APT system is shown in Fig. 1. The system consists of five optical paths - beacon receive optical path, beacon transmit optical path, signal transmit path, signal receive optical path and fine tracking path. The beacon path pointing accuracy will influence the target initial capture probability, the coaxiality of beacon path determines whether the coarse tracking link will be established. Portion of the signal laser (about 1/10 energy) will be splitted up for fine 
$\mathrm{T}$ a b l e. Explanation of acronyms.

\begin{tabular}{ll}
\hline Acronym & Definition \\
\hline APT & Acquisition, pointing and tracking \\
BSM & Beam splitter mirror \\
CCR & Cube corner reflector \\
COE & Coaxiality error \\
CTD & Coarse tracking detector \\
FM & Folding mirror \\
FSM & Fast steering mirror \\
FTD & Fine tracking detector \\
IFOV & Instantaneous field of view $(\mu \mathrm{rad})$ \\
PAM & Point ahead mirror \\
PE & Pointing error \\
RM & Reflector mirror \\
\hline
\end{tabular}

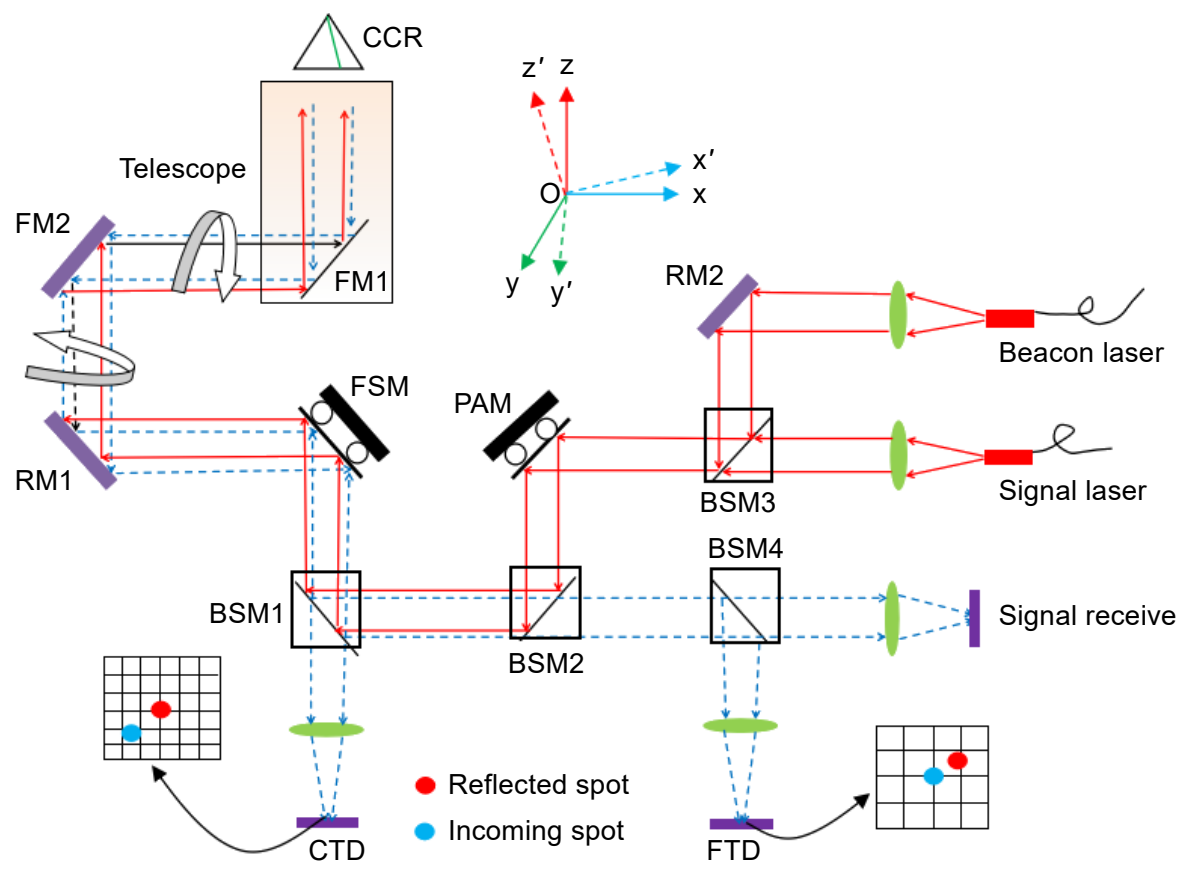

Fig. 1. Schematic view of the internal optical path structure of APT system. The beacon laser goes through the beam collimation system: RM2, BSM3, PAM, BSM2, BSM1, FSM, RM1, FM2 then retroreflected by CCR returns into the system and finally is focused on the CTD surface; IFOV of CTD is $6.1 \mu \mathrm{rad}$. The signal laser is partially retroreflected by CCR and goes inside the APT again and then is reflected by BSM4. Finally it is focused on the surface of the FTD and the IFOV is $4.4 \mu \mathrm{rad}$. So the coaxiality can be worked out according to the distance between the two spots centroids. The basic coordinate of the APT is $o-x y z$ and the coordinates of each optical element are parallel to $o-x y z$. If there exists an assembly error, the coordinate becomes $o-x^{\prime} y^{\prime} z^{\prime}$ and the new laser pointing vector can be derived out through the error transformation matrix. 
tracking, then if the incoming laser beam is to be stabilized on the detector the point ahead mirror (PAM) will control the outgoing signal laser point to the place where the counter satellite will be. So, the coaxiality between the signal transmit optical path and the fine tracking path determines whether the communication link will be established. Therefore, the pointing precision of each optical path and the coaxiality are very important factors which need quantitative analysis and provide theoretical basis for ground assembling.

\section{Assembly error model of reflector and axis}

\subsection{Reflector assembly error analysis}

Obviously, the assembly error (AE) of a reflector will contribute to the pointing error, but the internal optical path of APT system is intricate and the installation types of the reflectors are different. The PAM and FSM adopted the closed-loop control method which can realize error self-calibration but not the other reflectors. Figure 2 shows different installation types of reflectors. The first type - the normals of the two adjacent reflectors are parallel to each other and both of them are in the same plane. The second type - the normals are perpendicular to each other and are also in the same plane. The third type - the normals are neither parallel nor coplanar.
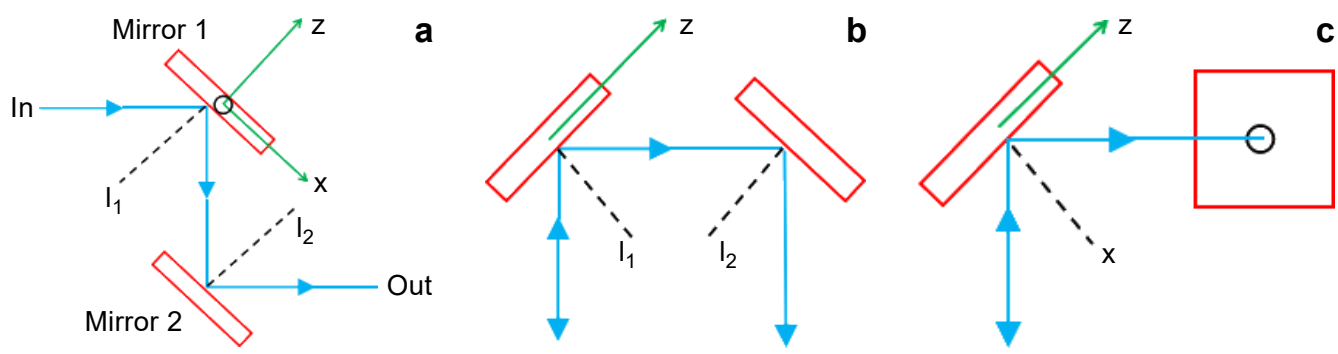

Fig. 2. Different reflector connection types. (a) $l_{1} \| l_{2}$ (b) $l_{1} \perp l_{2}$, and (c) neither parallel nor perpendicular. Where $l_{1}$ and $l_{2}$ are the normal of the two reflectors.

The reflection matrix of a reflector can be defined as follows:

$$
H=E-2 N N^{\mathrm{T}}
$$

where $N$ is the normal of the mirror and $E=[1,0,0 ; 0,1,0 ; 0,0,1]^{\mathrm{T}}$ is the unit matrix. Assuming that the reflector rotates $\varphi$ degree around any axis, then the normal direction of the reflector changes and the transfer matrices are:

$$
R_{x, \varphi}=\left[\begin{array}{lll}
1 & 0 & 0 \\
0 & \cos \varphi & \sin \varphi \\
0 & -\sin \varphi & \cos \varphi
\end{array}\right]
$$




$$
\begin{aligned}
& R_{y, \varphi}=\left[\begin{array}{lll}
\cos \varphi & 0 & -\sin \varphi \\
0 & 1 & 0 \\
\sin \varphi & 0 & \cos \varphi
\end{array}\right] \\
& R_{z, \varphi}=\left[\begin{array}{lll}
\cos \varphi & \sin \varphi & 0 \\
-\sin \varphi & \cos \varphi & 0 \\
0 & 0 & 1
\end{array}\right]
\end{aligned}
$$

where $R_{x, \varphi}, R_{y, \varphi}$ and $R_{z, \varphi}$ mean transformation matrices of the axis $x, y$ and $z$, respectively. The new normal of the reflector is as follows:

$$
N^{\prime}=R_{\varphi, z} R_{\varphi, y} R_{\varphi, x} N
$$

According to the formulas (1)-(3) we can obtain the new reflection matrix $H^{\prime}$

$$
H^{\prime}=E-2 N^{\prime} N^{\prime \mathrm{T}}=E-2 S_{\varphi, z} S_{\varphi, y} S_{\varphi, x} N^{\prime}\left(S_{\varphi, z} S_{\varphi, y} S_{\varphi, x} N^{\prime}\right)^{\mathrm{T}}
$$

Then, the pointing error induced by the reflector assembly error can be expressed as the angle between the vector $H_{1}$ and the vector $H$ as follows:

$$
\delta=\arccos \left\langle H_{1} \cdot H\right\rangle
$$

According to the model, the influence of installation types of the reflectors on the pointing error takes place and the results can be seen in Fig. 3.

As we all know, the reflected laser vector will not change if the reflector rotates around its normal, so we just take the rotate angle of axis $x$ and axis $y$ into consideration. Figure 3 a gives a clear relationship between the pointing vector and the reflector assembly error of axis $x$. The error of a type 3 is bigger than any other types. The pointing error

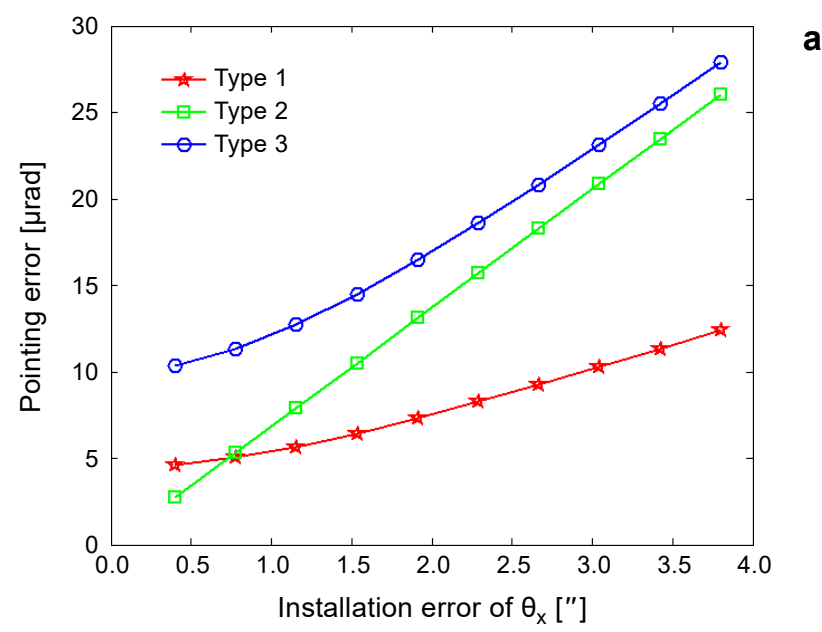

Fig. 3. Pointing error induced by the reflector assembly error of (a) axis $x$ and (b) axis $y$. 


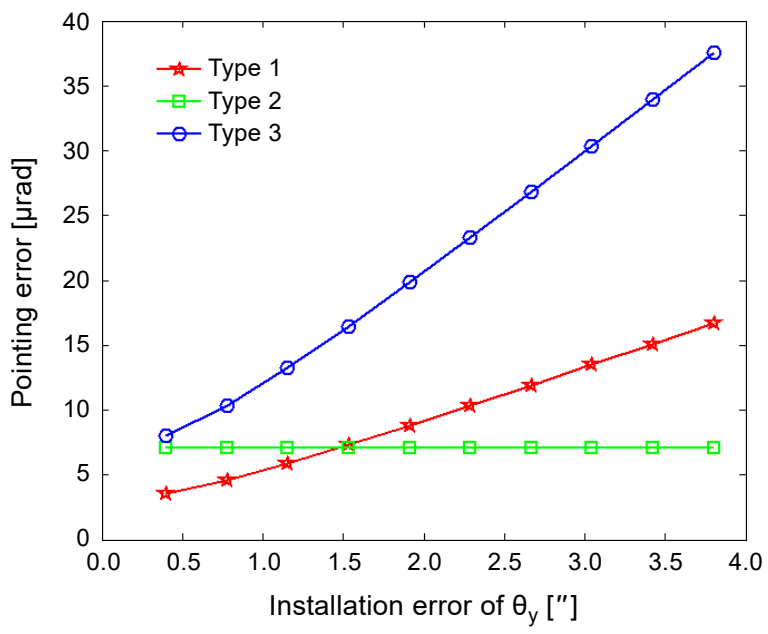

b

Fig. 3. Continued.

is basically linear with the assembly error $\varphi_{x}$, and if $\varphi_{x}<0.6^{\prime \prime}$ there will be $P_{2}<P_{1}$, otherwise if $\varphi_{x}>0.6^{\prime \prime}$ there will be $P_{2}>P_{1}$. Figure $3 \mathbf{b}$ shows the relationship between the pointing error and the assembly error of axis $y$. Similarly, the error of a type 3 is bigger than other cases but the value of $P_{2}$ is constant which is $7.5 \mu \mathrm{rad}$. Moreover if $\varphi_{y}<1.5^{\prime \prime}$ there will be $P_{1}>P_{2}$, otherwise $P_{1}<P_{2}$. So in the APT design process, we should avoid using the third installation method as far as it is possible.

\subsection{Axis assembly error}

The axis error can also contribute to the PE of APT system. The axis error is mainly induced by the axis assembly error and machining error [11]. The error is mainly manifested as radial sloshing which means not perpendicular to the installation surface. Figures $4 \mathbf{a}$ and $4 \mathbf{b}$ show the axis error of elevation axis and azimuth axis, assuming
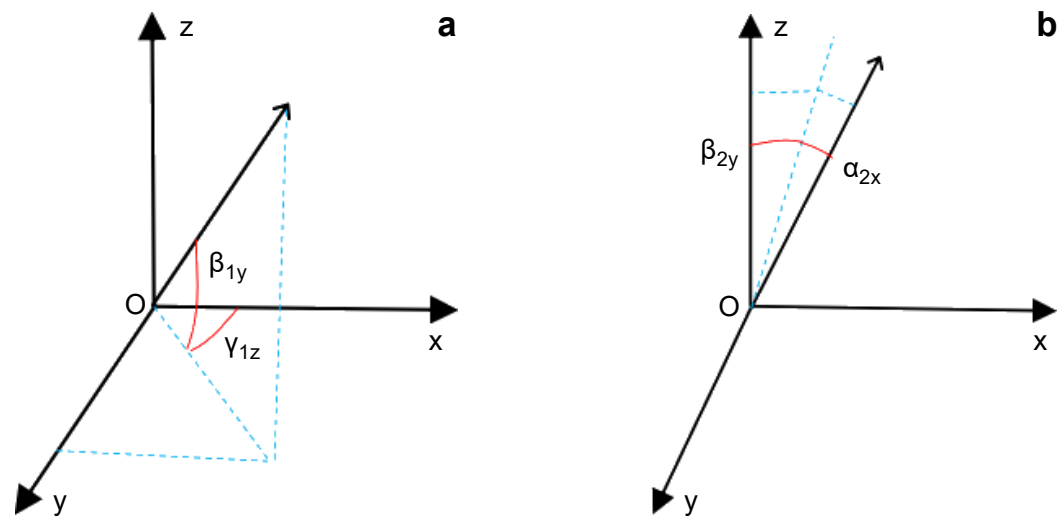

Fig. 4. Axis assembly error of (a) azimuth axis and (b) elevation axis. 
that the number of azimuth axes is 1 and the basic axis direction is $x$, the number of elevation axes is 2 and the basic direction is $z$. If there exists an assembly error, the actual beam direction of the azimuth axis can be expressed as the original vector rotates $\gamma_{1 z}$ degree around axis $z$ and $\beta_{1 y}$ degree around axis $y$. The actual pointing vector of the azimuth axis can be expressed as the original axis vector rotates $\gamma_{2 z}$ degree around axis $z$ and $\beta_{2 y}$ degree around axis $y$.

In previous studies, the authors discussed the detection method of PE and COE, but the pointing error induced by the assembly error has not been discussed. In order to improve the design quality of the APT system, it is necessary to make a quantitative analysis of the influence of the assembly error on PE and COE. The coaxiality is determined by the vector angle of the outgoing beam $A^{\prime}$ and the incoming beam $A$, and the pointing direction changed with the axis rotation angle. The PE is also related to the reflective order of the beam in the APT. Take the outgoing beam for example, assuming that the rotation angle of the azimuth axis is $\theta_{\mathrm{az}, z}$ and the rotation angle of the elevation axis is $\theta_{\mathrm{el}, z}$, the outgoing laser transmission process of the beam in the axis is as follows: azimuth axis $\rightarrow$ FM $2 \rightarrow$ elevation axis $\rightarrow$ FM1 $\rightarrow$ exit. Then, we can write out the error transformation formula:

$A_{\mathrm{out}}^{\prime}=R\left(\theta_{\mathrm{el}, x}\right) R\left(\theta_{\mathrm{az}, z}\right) T_{\mathrm{el}} R^{-1}\left(\theta_{\mathrm{el}, x}\right) R^{-1}\left(\theta_{\mathrm{az}, z}\right) R_{\mathrm{el}} R\left(\theta_{\mathrm{az}, z}\right) T_{\mathrm{az}} R^{-1}\left(\theta_{\mathrm{az}, z}\right) R_{\mathrm{az}} \bullet A_{\mathrm{out}}$

where $T_{\mathrm{el}}$ and $T_{\mathrm{az}}$ are the unit matrices

$$
T_{\mathrm{el}}=T_{\mathrm{az}}=\left[\begin{array}{lll}
1 & & \\
& 1 & \\
& & \\
& & 1
\end{array}\right]
$$

while $R_{\mathrm{az}}$ and $R_{\mathrm{el}}$ denotes the axis error transmit matrix which can be derived out according to formula (2):

$$
\begin{aligned}
& R_{\mathrm{az}}=S\left(\beta_{2}\right) S\left(\gamma_{2}\right) \\
& R_{\mathrm{el}}=S\left(\beta_{1}\right) S\left(\gamma_{1}\right)
\end{aligned}
$$

And the incoming laser beam transfer process is: FM1 $\rightarrow$ elevation axis $\rightarrow$ FM2 $\rightarrow$ azimuth axis, then we can write out the vector transfer process:

$$
A_{\mathrm{in}}^{\prime}=R\left(\theta_{\mathrm{az}, z}\right) R\left(\theta_{\mathrm{el}, x}\right) T_{\mathrm{az}} R^{-1}\left(\theta_{\mathrm{az}, z}\right) R^{-1}\left(\theta_{\mathrm{el}, x}\right) R_{\mathrm{az}} R\left(\theta_{\mathrm{el}, x}\right) T_{\mathrm{az}} R^{-1}\left(\theta_{\mathrm{el}, x}\right) R_{\mathrm{el}} \bullet A_{\mathrm{in}}
$$

\section{Pointing error and coaxiality simulation analysis}

\subsection{The beacon transmit optical path}

According to Fig. 2, the laser transfer process of the outgoing beacon laser in APT is as follows: $\mathrm{RM} 2 \rightarrow \mathrm{BSM} 3 \rightarrow \mathrm{PAM} \rightarrow \mathrm{BSM} 2 \rightarrow \mathrm{BSM} 1 \rightarrow \mathrm{FSM} \rightarrow \mathrm{RM} 1 \rightarrow \mathrm{FM} 2 \rightarrow \mathrm{FM}$. 
Assuming that the vector of the outgoing beacon from the laser source is $A_{1}=[1,0,0]^{\mathrm{T}}$, according to formulas (2), (4) and (6), the vector of the beacon laser at the antenna surface is as follows:

$$
\begin{aligned}
A_{1}^{\prime}= & R\left(\theta_{\mathrm{el}, x}\right) R\left(\theta_{\mathrm{az}, z}\right) T_{\mathrm{el}} R^{-1}\left(\theta_{\mathrm{az}, z}\right) R^{-1}\left(\theta_{\mathrm{el}, x}\right) R_{\mathrm{el}} R\left(\theta_{\mathrm{az}, z}\right) T_{\mathrm{az}} R^{-1}\left(\theta_{\mathrm{az}, z}\right) \\
& \times R_{\mathrm{az}} H_{\mathrm{RM} 1} H_{\mathrm{FSM}} H_{\mathrm{BSM} 1} H_{\mathrm{BSM} 2} H_{\mathrm{PAM}} H_{\mathrm{BSM} 3} H_{\mathrm{RM} 2} A_{1} \\
= & R\left(\theta_{\mathrm{el}, x}\right) R\left(\theta_{\mathrm{az}, z}\right) T_{\mathrm{el}} R^{-1}\left(\theta_{\mathrm{az}, z}\right) R^{-1}\left(\theta_{\mathrm{el}, x}\right) R_{\mathrm{el}} R\left(\theta_{\mathrm{az}, z}\right) T_{\mathrm{az}} R^{-1}\left(\theta_{\mathrm{az}, z}\right) R_{\mathrm{az}} \\
& \times\left(E-2 N_{\mathrm{RM} 1} N_{\mathrm{RM} 1}^{\mathrm{T}}\right) H_{\mathrm{FSM}}\left(E-2 N_{\mathrm{BSM} 1} N_{\mathrm{BSM} 1}^{\mathrm{T}}\right)\left(E-2 N_{\mathrm{BSM} 2} N_{\mathrm{BSM} 2}^{\mathrm{T}}\right) H_{\mathrm{PAM}} \\
& \times\left(E-2 N_{\mathrm{BSM} 3} N_{\mathrm{BSM} 3}^{\mathrm{T}}\right)\left(E-2 N_{\mathrm{RM} 2} N_{\mathrm{RM} 2}^{\mathrm{T}}\right) A_{1} \\
= & R\left(\theta_{\mathrm{el}, x}\right) R\left(\theta_{\mathrm{az}, z}\right) T_{\mathrm{el}} R^{-1}\left(\theta_{\mathrm{az}, z}\right) R^{-1}\left(\theta_{\mathrm{el}, x}\right) \\
& \times S\left(\beta_{1}\right) S\left(\gamma_{1}\right) R\left(\theta_{\mathrm{az}, z}\right) T_{\mathrm{az}} R^{-1}\left(\theta_{\mathrm{az}, z}\right) S\left(\beta_{2}\right) S\left(\gamma_{2}\right) \\
& \times\left[E-2 S_{\gamma z, \mathrm{RM} 1} S_{\beta y, \mathrm{RM} 1} N_{\mathrm{RM} 1}\left(S_{\gamma z, \mathrm{RM} 1} S_{\beta y, \mathrm{RM} 1} N_{\mathrm{RM} 1}\right)^{\mathrm{T}}\right] H_{\mathrm{FSM}} \\
& \times\left[E-2 S_{\gamma z, \mathrm{BSM} 1} S_{\beta y, \mathrm{BSM} 1} N_{\mathrm{BSM} 1}\left(S_{\gamma z, \mathrm{BSM} 1} S_{\beta y, \mathrm{BSM} 1} N_{\mathrm{BSM} 1}\right)^{\mathrm{T}}\right] \\
& \times\left[E-2 S_{\gamma z, \mathrm{BSM} 2} S_{\beta y, \mathrm{BSM} 2} N_{\mathrm{BSM} 2}\left(S_{\gamma z, \mathrm{BSM} 2} S_{\beta y, \mathrm{BSM} 2} N_{\mathrm{BSM} 2}\right)^{\mathrm{T}}\right] H_{\mathrm{PAM}} \\
& \times\left[E-2 S_{\gamma z, \mathrm{BSM} 3} S_{\beta y, \mathrm{BSM} 3} N_{\mathrm{BSM} 3}\left(S_{\gamma z, \mathrm{BSM} 3} S_{\beta y, \mathrm{BSM} 3} N_{\mathrm{BSM} 3}\right)^{\mathrm{T}}\right] \\
& \times\left[E-2 S_{\gamma z, \mathrm{RM} 2} S_{\beta y, \mathrm{RM} 2} N_{\mathrm{RM} 2}\left(S_{\gamma z, \mathrm{RM} 2} S_{\beta y, \mathrm{RM} 2} N_{\mathrm{RM} 2}\right)^{\mathrm{T}}\right] A_{1}
\end{aligned}
$$

where $H_{(\cdot)}$ denotes the reflective matrix of the corresponding reflector, $N_{(\cdot)}$ denotes the corresponding normal. If there is no assembly error, the beam pointing vector can be rewritten as:

$$
\begin{aligned}
A_{1}^{\prime \prime}= & R\left(\theta_{\mathrm{el}, x}\right) R\left(\theta_{\mathrm{az}, z}\right) T_{\mathrm{el}} R^{-1}\left(\theta_{\mathrm{az}, z}\right) R^{-1}\left(\theta_{\mathrm{el}, x}\right) R_{\mathrm{el}} R\left(\theta_{\mathrm{az}, z}\right) T_{\mathrm{az}} R^{-1}\left(\theta_{\mathrm{az}, z}\right) \\
& \times R_{\mathrm{az}} h_{\mathrm{RM} 1} h_{\mathrm{FSM}} h_{\mathrm{BSM} 1} h_{\mathrm{BSM} 2} h_{\mathrm{PAM}} h_{\mathrm{BSM} 3} h_{\mathrm{RM} 2} A_{1}
\end{aligned}
$$

where $h_{(\cdot)}$ denotes the transfer matrix of the reflectors without the assembly error. Then, according to formulas (11) and (12), the pointing error of the beacon emit path can be expressed as

$$
\delta_{1}=\arccos \left(\frac{A_{1}^{\prime} \cdot A_{1}^{\prime \prime}}{\left|A_{1}^{\prime}\right|\left|A_{1}^{\prime \prime}\right|}\right)
$$




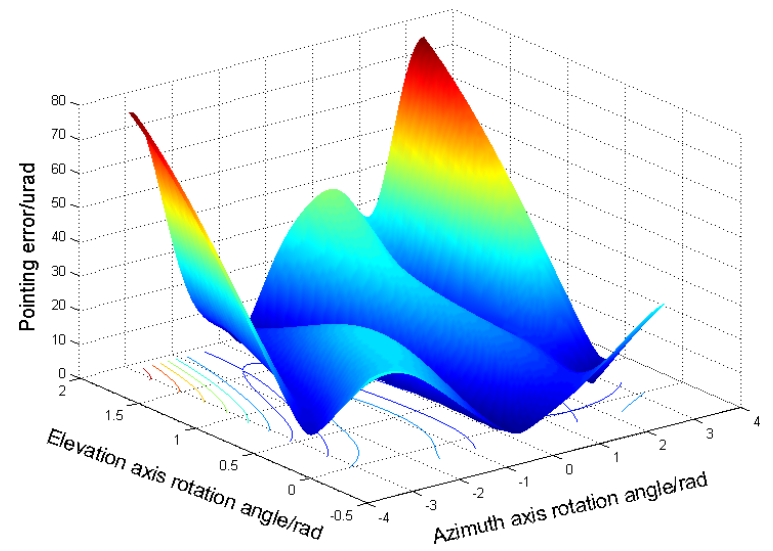

a

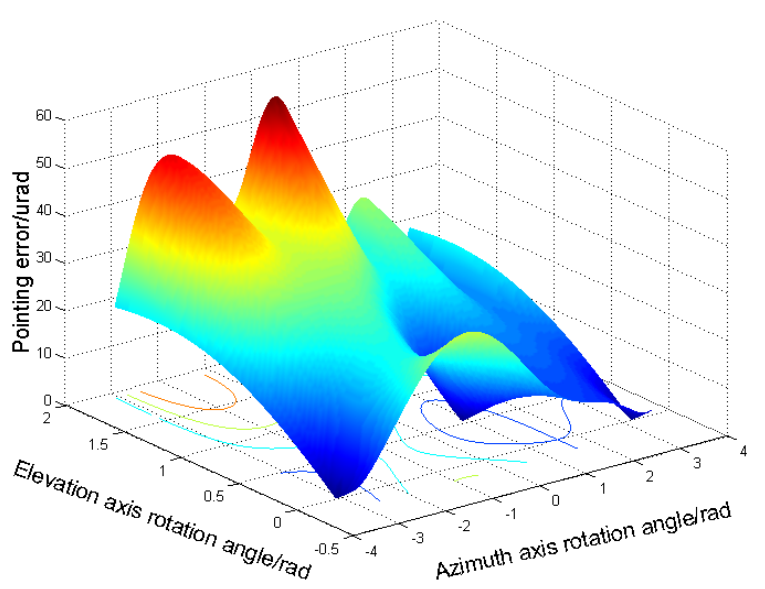

b

Fig. 5. Pointing error of (a) $\delta_{1}=2^{\prime \prime}, \delta_{2}=5^{\prime \prime}$, and (b) $\delta_{1}=2^{\prime \prime}, \delta_{2}=-5^{\prime \prime}$. Where $\delta_{1}$ denotes the axis error and $\delta_{2}$ denotes the reflector error.

In Fig. 5, the beacon emit optical path pointing error is performed. According to the current assembling technology, we can assume that the axis assembly error used in this paper is $2^{\prime \prime}$ and the reflector assembly error is $5^{\prime \prime}[\underline{4}, \underline{12}]$. The axis rotate angle of the azimuth axis $\theta_{\mathrm{az}}$ is $-180^{\circ}$ to $180^{\circ}$ and the elevation angle $\theta_{\mathrm{el}}$ changes from $-10^{\circ}$ to $110^{\circ}$.

\subsection{The coarse tracking path $P E$ analysis}

The beam's travel order of the coarse tracking optical path (also means the beacon receive path) is as follows: incoming laser $\rightarrow$ FM1 $\rightarrow$ FM2 $\rightarrow$ RM1 $\rightarrow$ FSM $\rightarrow$ CTD, then according to formulas (2), (4) and (10), the pointing error of the coarse tracking optical path induced by the assembling error can be expressed as

$$
\begin{aligned}
A_{1}^{\prime}= & H_{\mathrm{FSM}} H_{\mathrm{RM} 1} R\left(\theta_{\mathrm{az}, z}\right) R\left(\theta_{\mathrm{el}, x}\right) T_{\mathrm{az}} R^{-1}\left(\theta_{\mathrm{az}, z}\right) R^{-1}\left(\theta_{\mathrm{el}, x}\right) \\
& \times R_{\mathrm{az}} R\left(\theta_{\mathrm{el}, x}\right) T_{\mathrm{el}} R^{-1}\left(\theta_{\mathrm{el}, x}\right) R_{\mathrm{el}} A_{05}
\end{aligned}
$$




$$
\begin{aligned}
= & H_{\mathrm{FSM}}\left(E-2 N_{\mathrm{RM} 1} N_{\mathrm{RM} 1}^{\mathrm{T}}\right) R\left(\theta_{\mathrm{az}, z}\right) R\left(\theta_{\mathrm{el}, x}\right) T_{\mathrm{az}} R^{-1}\left(\theta_{\mathrm{az}, z}\right) R^{-1}\left(\theta_{\mathrm{el}, x}\right) \\
& \times R_{\mathrm{az}} R\left(\theta_{\mathrm{el}, x}\right) T_{\mathrm{el}} R^{-1}\left(\theta_{\mathrm{el}, x}\right) R_{\mathrm{el}} A_{2}
\end{aligned}
$$

And the beam pointing vector without the assembly error is

$$
\begin{aligned}
A_{1}^{\prime \prime}= & H_{4} R\left(\theta_{\mathrm{az}, z}\right) R\left(\theta_{\mathrm{el}, x}\right) T_{\mathrm{az}} R^{-1}\left(\theta_{\mathrm{az}, z}\right) R^{-1}\left(\theta_{\mathrm{el}, x}\right) \\
& \times R_{\mathrm{az}} R\left(\theta_{\mathrm{el}, x}\right) T_{\mathrm{el}} R^{-1}\left(\theta_{\mathrm{el}, x}\right) R_{\mathrm{el}} A_{05} \\
= & h_{\mathrm{FSM}} h_{\mathrm{RM} 1}\left(E-2 N_{\mathrm{RM} 1} N_{\mathrm{RM} 1}^{\mathrm{T}}\right) R\left(\theta_{\mathrm{az}, z}\right) R\left(\theta_{\mathrm{el}, x}\right) T_{\mathrm{az}} R^{-1}\left(\theta_{\mathrm{az}, z}\right) R^{-1}\left(\theta_{\mathrm{el}, x}\right) \\
& \times R_{\mathrm{az}} R\left(\theta_{\mathrm{el}, x}\right) T_{\mathrm{el}} R^{-1}\left(\theta_{\mathrm{el}, x}\right) R_{\mathrm{el}} A_{2}
\end{aligned}
$$

The pointing error of the coarse tracking path is shown in Fig. 6. The results show that the maximum value of PE occurred when $\theta_{\mathrm{az}}=0^{\circ}$ and $\theta_{\mathrm{el}}=90^{\circ}$ which is usually called the zenith blind zone.

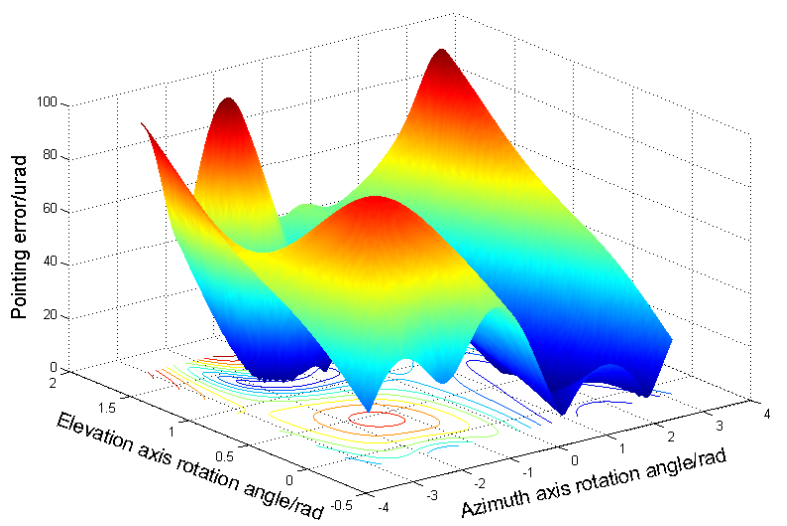

a

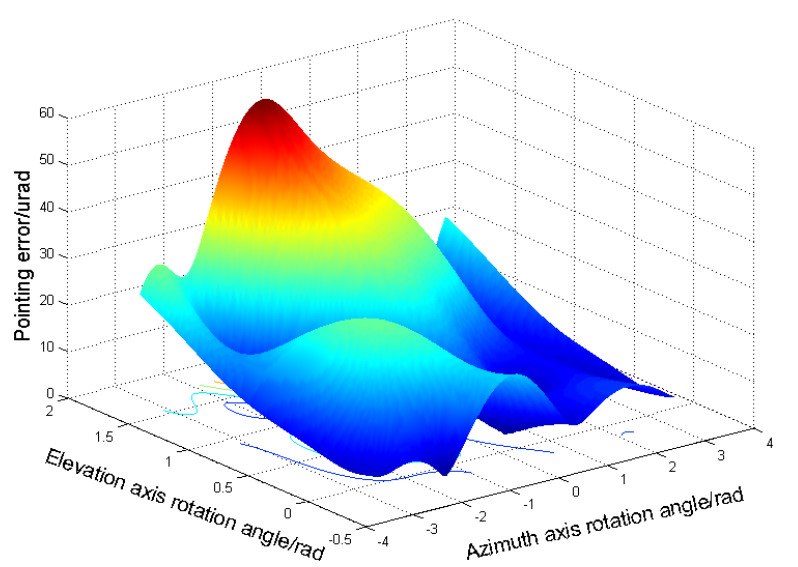

b

Fig. 6. The PE of the coarse tracking optical path (a) $\delta_{1}=2^{\prime \prime}, \delta_{2}=5^{\prime \prime}$, and (b) $\delta_{1}=2^{\prime \prime}, \delta_{2}=-5^{\prime \prime}$. 


\subsection{The coaxiality analysis of beacon path}

The beacon laser sent out from the APT and then reflected into the APT by CCR (cube corner reflector) finally arrives at the surface of CTD; according to formulas (11) and (14), the beam point vector with the assembly error can be written as

$$
\begin{aligned}
A_{\text {beacon }}^{\prime}= & H_{\mathrm{FSM}} H_{\mathrm{RM} 1} R\left(\theta_{\mathrm{az}, z}\right) R\left(\theta_{\mathrm{el}, x}\right) T_{\mathrm{az}} R^{-1}\left(\theta_{\mathrm{az}, z}\right) R^{-1}\left(\theta_{\mathrm{el}, x}\right) \\
& \times R_{\mathrm{az}} R\left(\theta_{\mathrm{el}, x}\right) T_{\mathrm{el}} R^{-1}\left(\theta_{\mathrm{el}, x}\right) R_{\mathrm{el}} \bullet R\left(\theta_{\mathrm{el}, x}\right) R\left(\theta_{\mathrm{az}, z}\right) T_{\mathrm{el}} \\
& \times R^{-1}\left(\theta_{\mathrm{az}, z}\right) R^{-1}\left(\theta_{\mathrm{el}, x}\right) R_{\mathrm{el}} R\left(\theta_{\mathrm{az}, z}\right) T_{\mathrm{az}} R^{-1}\left(\theta_{\mathrm{az}, z}\right) R_{\mathrm{az}} \\
& \times H_{\mathrm{RM} 1} H_{\mathrm{FSM}} H_{\mathrm{BSM} 1} H_{\mathrm{BSM} 2} H_{\mathrm{PAM}} H_{\mathrm{BSM} 3} H_{\mathrm{RM} 2} A_{\text {beacon }}
\end{aligned}
$$

According to formulas (12) and (15), the beam pointing vector without the assembly error is

$$
\begin{aligned}
A_{\text {beacon }}^{\prime \prime}= & H_{\mathrm{FSM}} h_{\mathrm{RM} 1} R\left(\theta_{\mathrm{az}, z}\right) R\left(\theta_{\mathrm{el}, x}\right) T_{\mathrm{az}} R^{-1}\left(\theta_{\mathrm{az}, z}\right) R^{-1}\left(\theta_{\mathrm{el}, x}\right) \\
& \times R_{\mathrm{az}} R\left(\theta_{\mathrm{el}, x}\right) T_{\mathrm{el}} R^{-1}\left(\theta_{\mathrm{el}, x}\right) R_{\mathrm{el}} \bullet R\left(\theta_{\mathrm{el}, x}\right) R\left(\theta_{\mathrm{az}, z}\right) T_{\mathrm{el}} \\
& \times R^{-1}\left(\theta_{\mathrm{az}, z}\right) R^{-1}\left(\theta_{\mathrm{el}, x}\right) R_{\mathrm{el}}^{\prime} R\left(\theta_{\mathrm{az}, z}\right) T_{\mathrm{az}} R^{-1}\left(\theta_{\mathrm{az}, z}\right) R_{\mathrm{az}}^{\prime} \\
& \times h_{\mathrm{RM} 1} h_{\mathrm{FSM}} h_{\mathrm{BSM} 1} h_{\mathrm{BSM} 2} h_{\mathrm{PAM}} h_{\mathrm{BSM} 3} h_{\mathrm{RM} 2} A_{\text {beacon }}
\end{aligned}
$$

The coaxiality of the beacon path is the angle between vector $A_{\text {beacon }}^{\prime}$ and vector

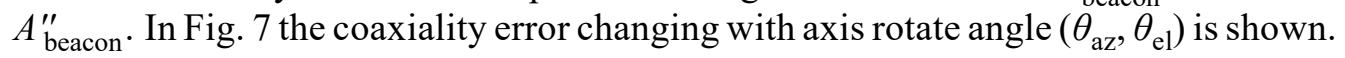
Compare Fig. 7a with Fig. 7c we can found that the AE has no influence on COE distribution but has an influence on the magnitude of COE, and the smaller of the sum of

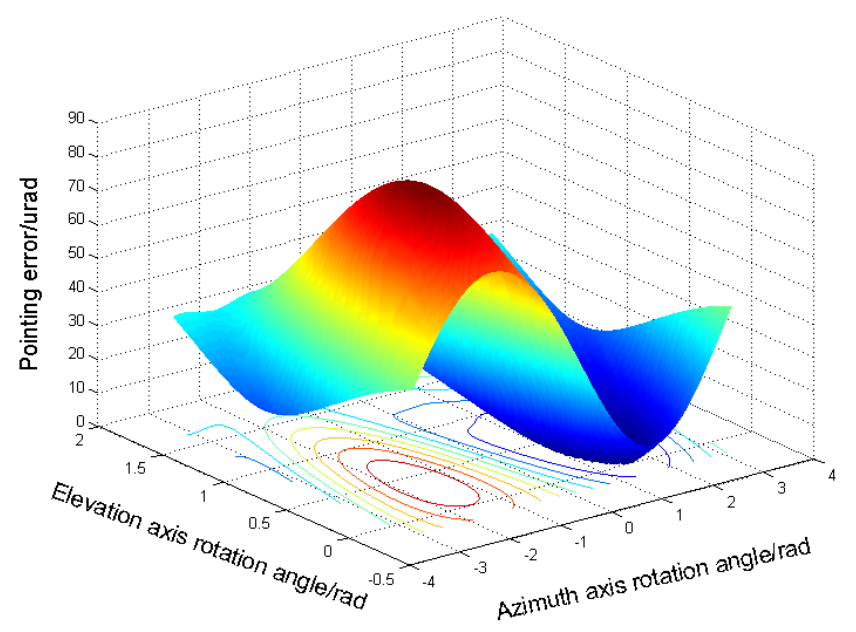

a

Fig. 7. Coaxiality simulation results of the beacon path. (a) $\delta_{1}=2^{\prime \prime}, \delta_{2}=5^{\prime \prime}$, and (b) error frequency histogram; (c) $\delta_{1}=2^{\prime \prime}, \delta_{2}=-5^{\prime \prime}$, and (d) error frequency histogram. 


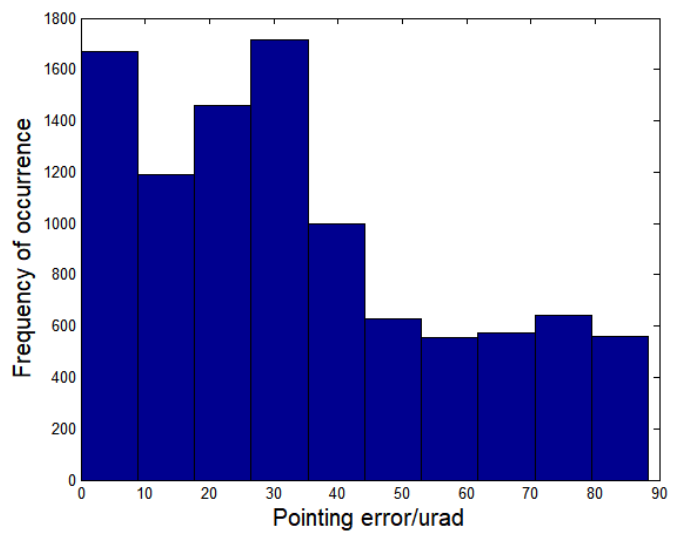

b

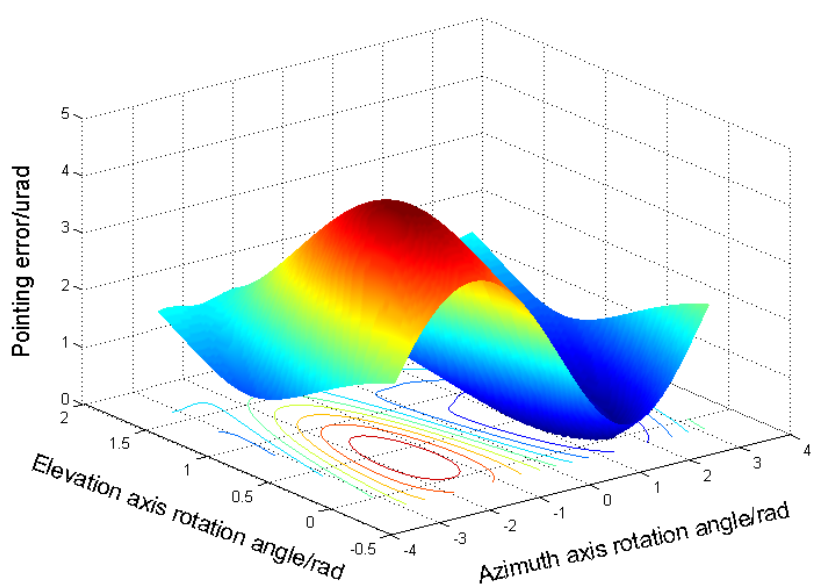

C

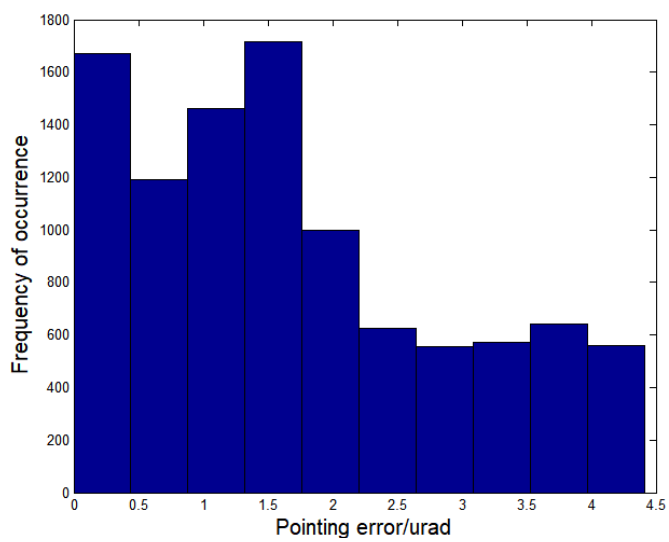

d

Fig. 7. Continued. 
axis assembly error and reflector assembly error, the smaller of COE this is due to error cancellation. The maximum COE occurred at $\theta_{\mathrm{az}}=-1.8 \mathrm{rad}$ and $\theta_{\mathrm{el}}=0.4 \mathrm{rad}$.

\subsection{The signal transmit path $P E$ analysis}

The beam's travel order of the signal transmit optical path is as follows: outgoing beam $\rightarrow$ BSM3 $\rightarrow$ PAM $\rightarrow$ BSM2 $\rightarrow$ BSM1 $\rightarrow$ FSM $\rightarrow$ RM1 $\rightarrow$ FM2 $\rightarrow$ FM1. Then the assembly error induced pointing error vector can be derived out:

$$
\begin{aligned}
A_{3}^{\prime}= & R\left(\theta_{\mathrm{el}, x}\right) R\left(\theta_{\mathrm{az}, z}\right) T_{\mathrm{el}} R^{-1}\left(\theta_{\mathrm{az}, z}\right) R^{-1}\left(\theta_{\mathrm{el}, x}\right) R_{\mathrm{el}} R\left(\theta_{\mathrm{az}, z}\right) T_{\mathrm{az}} R^{-1}\left(\theta_{\mathrm{az}, z}\right) R_{\mathrm{az}} \\
& \times H_{\mathrm{RM} 1} H_{\mathrm{FSM}} H_{\mathrm{BSM} 1} H_{\mathrm{BSM} 2} H_{\mathrm{PAM}} A_{3}
\end{aligned}
$$

And the outgoing beam vector without the pointing error is:

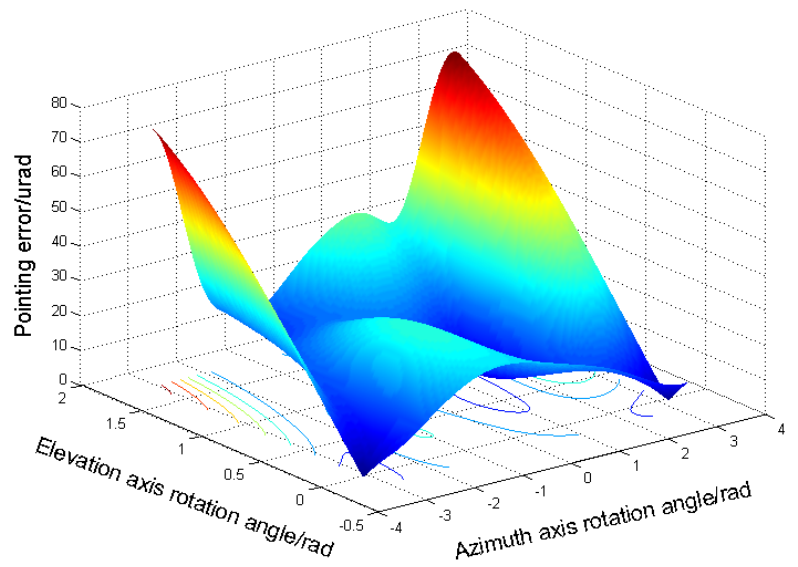

a

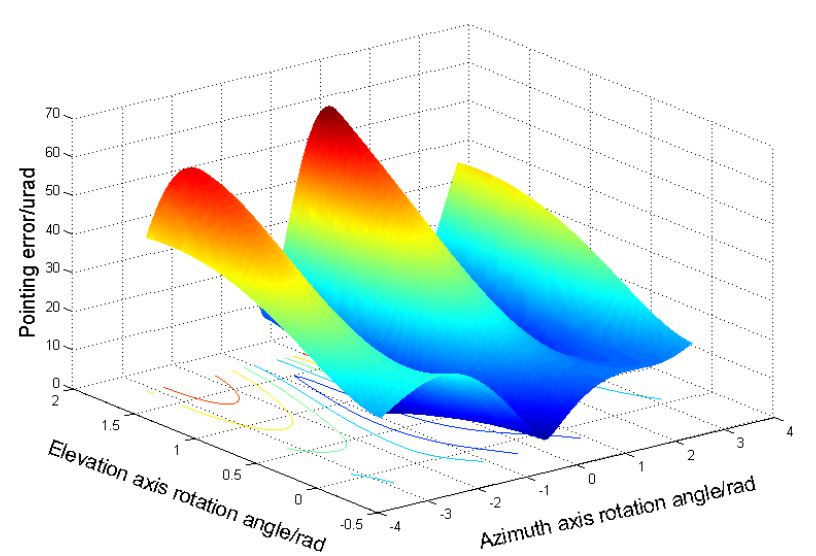

b

Fig. 8. Signal transmit optical path PE simulation results of (a) $\delta_{1}=2^{\prime \prime}, \delta_{2}=5^{\prime \prime}$, and (b) $\delta_{1}=2^{\prime \prime}, \delta_{2}=-5^{\prime \prime}$. 


$$
\begin{aligned}
A_{3}^{\prime \prime}= & R\left(\theta_{\mathrm{el}, x}\right) R\left(\theta_{\mathrm{az}, z}\right) T_{\mathrm{el}} R^{-1}\left(\theta_{\mathrm{az}, z}\right) R^{-1}\left(\theta_{\mathrm{el}, x}\right) R_{\mathrm{el}} R\left(\theta_{\mathrm{az}, z}\right) T_{\mathrm{az}} R^{-1}\left(\theta_{\mathrm{az}, z}\right) R_{\mathrm{az}} \\
& \times h_{\mathrm{RM} 1} h_{\mathrm{FSM}} h_{\mathrm{BSM} 1} h_{\mathrm{BSM} 2} h_{\mathrm{PAM}} A_{3}
\end{aligned}
$$

The PE simulation of the signal transmit optical path is performed and the result is shown in Fig. 8. Taking different sizes of errors into consideration, we can find that the PE distribution of the signal path is different from that of the beacon path, and the $\mathrm{PE}$ varies with the sum of the axis assembly error and the reflector assembly error, if we take the axis error and the reflector error, the same sign of the error is symmetric with the azimuth axis, and when $\theta_{\mathrm{az}}= \pm 180^{\circ}, \theta_{\mathrm{el}}=+90^{\circ}$ the $\mathrm{PE}$ reaches peak.

\subsection{The fine tracking optical path $P E$ analysis}

In the same way, the incoming laser of the fine tracking path travel process can be expressed as

$$
\begin{aligned}
A_{4}^{\prime}= & H_{\mathrm{BSM} 4} H_{\mathrm{BSM} 1} H_{\mathrm{FSM}} H_{\mathrm{RM} 1} R\left(\theta_{\mathrm{az}, z}\right) R\left(\theta_{\mathrm{el}, x}\right) T_{\mathrm{az}} R^{-1}\left(\theta_{\mathrm{az}, z}\right) R^{-1}\left(\theta_{\mathrm{el}, x}\right) \\
& \times R_{\mathrm{az}} R\left(\theta_{\mathrm{el}, x}\right) T_{\mathrm{el}} R^{-1}\left(\theta_{\mathrm{el}, x}\right) R_{\mathrm{el}} A_{4}
\end{aligned}
$$

And the pointing beam vector without the assembly error is:

$$
\begin{aligned}
A_{4}^{\prime \prime}= & h_{\mathrm{BSM} 4} h_{\mathrm{BSM} 1} h_{\mathrm{FSM}} h_{\mathrm{RM} 1} R\left(\theta_{\mathrm{az}, z}\right) R\left(\theta_{\mathrm{el}, x}\right) T_{\mathrm{az}} R^{-1}\left(\theta_{\mathrm{az}, z}\right) R^{-1}\left(\theta_{\mathrm{el}, x}\right) \\
& \times R_{\mathrm{az}} R\left(\theta_{\mathrm{el}, x}\right) T_{\mathrm{el}} R^{-1}\left(\theta_{\mathrm{el}, x}\right) R_{\mathrm{el}} A_{4}
\end{aligned}
$$

Taking the same size of the assembly error into consideration, the PE of the fine tracking optical path varies regularly with axis rotate angle simulation results is shown in Fig. 9. The assembly errors have influence both on distribution and magnitude of $\mathrm{PE}$, and the maximum value occurred when $\theta_{\mathrm{az}}= \pm 180^{\circ}$ and $\theta_{\mathrm{el}}=+90^{\circ}$ in Fig. 9a and $\theta_{\mathrm{az}}=0^{\circ}, \theta_{\mathrm{el}}=+90^{\circ}$ in Fig. $9 \mathbf{b}$.

\subsection{The coaxiality analysis of signal path}

The signal laser delivered out from the APT and then reflected by the CCR, finally focused on the surface of FTD. According to formulas (18) and (20), the beam pointing vector of the signal optical path can be written as

$$
\begin{aligned}
A_{\text {signal }}^{\prime}= & H_{\mathrm{BSM} 4} H_{\mathrm{BSM} 1} H_{\mathrm{FSM}} H_{\mathrm{RM} 1} R\left(\theta_{\mathrm{az}, z}\right) R\left(\theta_{\mathrm{el}, x}\right) T_{\mathrm{az}} R^{-1}\left(\theta_{\mathrm{az}, z}\right) R^{-1}\left(\theta_{\mathrm{el}, x}\right) R_{\mathrm{az}} \\
& \times R\left(\theta_{\mathrm{el}, x}\right) T_{\mathrm{el}} R^{-1}\left(\theta_{\mathrm{el}, x}\right) R_{\mathrm{el}} \bullet R\left(\theta_{\mathrm{el}, x}\right) R\left(\theta_{\mathrm{az}, z}\right) T_{\mathrm{el}} R^{-1}\left(\theta_{\mathrm{az}, z}\right) R^{-1}\left(\theta_{\mathrm{el}, x}\right) \\
& \times R_{\mathrm{el}} R\left(\theta_{\mathrm{az}, z}\right) T_{\mathrm{az}} R^{-1}\left(\theta_{\mathrm{az}, z}\right) R_{\mathrm{az}} H_{\mathrm{RM} 1} H_{\mathrm{FSM}} H_{\mathrm{BSM} 1} H_{\mathrm{BSM} 2} H_{\mathrm{PAM}} A_{\mathrm{signal}}
\end{aligned}
$$




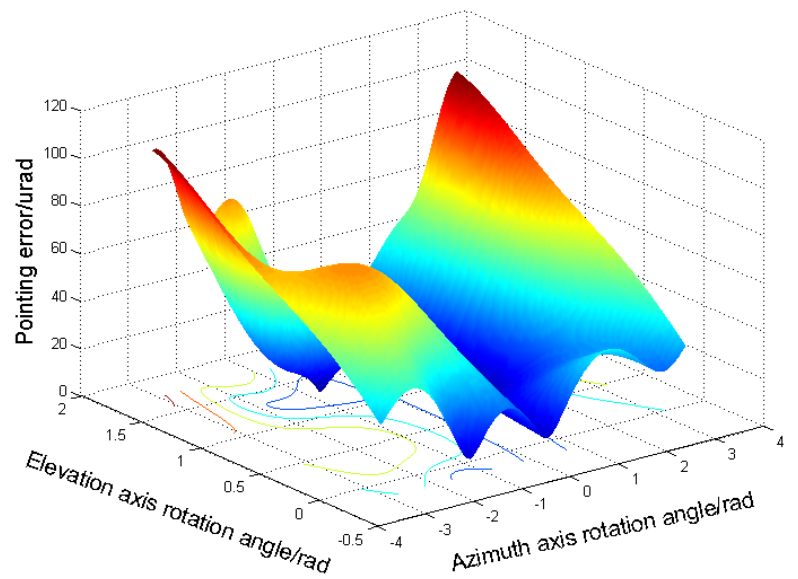

a

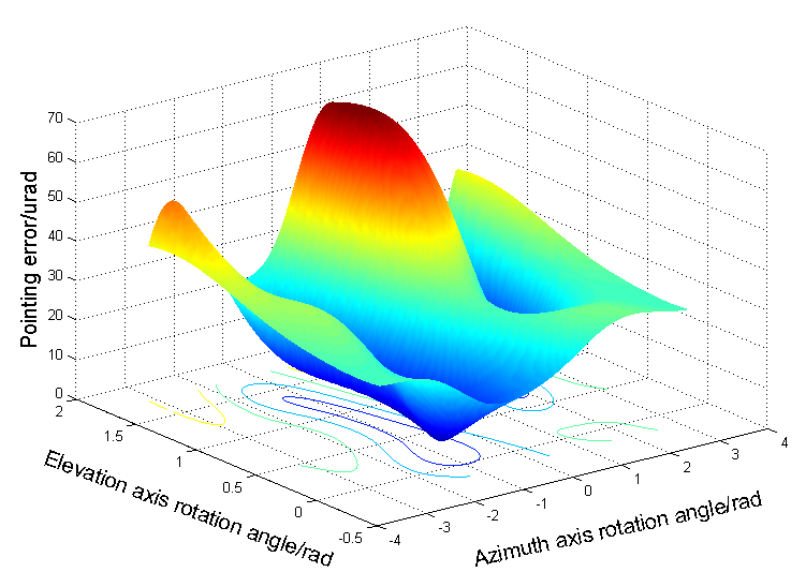

b

Fig. 9. Simulation results of fine tracking optical path of (a) $\delta_{1}=2^{\prime \prime}, \delta_{2}=5^{\prime \prime}$, and (b) $\delta_{1}=2^{\prime \prime}, \delta_{2}=-5^{\prime \prime}$.

And the beam pointing vector without the assembly error can be expressed as

$$
\begin{aligned}
A_{\text {signal }}^{\prime \prime \prime}= & h_{\mathrm{BSM} 4} h_{\mathrm{BSM} 1} h_{\mathrm{FSM}} h_{\mathrm{RM} 1} R\left(\theta_{\mathrm{az}, z}\right) R\left(\theta_{\mathrm{el}, x}\right) T_{\mathrm{az}} R^{-1}\left(\theta_{\mathrm{az}, z}\right) R^{-1}\left(\theta_{\mathrm{el}, x}\right) R_{\mathrm{az}} \\
& \times R\left(\theta_{\mathrm{el}, x}\right) T_{\mathrm{el}} R^{-1}\left(\theta_{\mathrm{el}, x}\right) R_{\mathrm{el}} \bullet R\left(\theta_{\mathrm{el}, x}\right) R\left(\theta_{\mathrm{az}, z}\right) T_{\mathrm{el}} R^{-1}\left(\theta_{\mathrm{az}, z}\right) R^{-1}\left(\theta_{\mathrm{el}, x}\right) \\
& \times R_{\mathrm{el}} R\left(\theta_{\mathrm{az}, z}\right) T_{\mathrm{az}} R^{-1}\left(\theta_{\mathrm{az}, z}\right) R_{\mathrm{az}} h_{\mathrm{RM} 1} h_{\mathrm{FSM}} h_{\mathrm{BSM} 1} h_{\mathrm{BSM} 2} h_{\mathrm{PAM}} A_{\text {signal }}
\end{aligned}
$$

Then the coaxiality of signal receive path and fine tracking path can be expressed as

$$
\delta_{\text {signal }}=\arccos \left(\frac{A_{\text {signal }}^{\prime} \cdot A_{\text {signal }}^{\prime \prime}}{\left|A_{\text {signal }}^{\prime}\right|\left|A_{\text {signal }}^{\prime \prime}\right|}\right)
$$

The coaxiality simulation of the signal optical path has been performed and the results can be seen in Fig 10. The results from Figs. 10a and 10c show that the COE of 


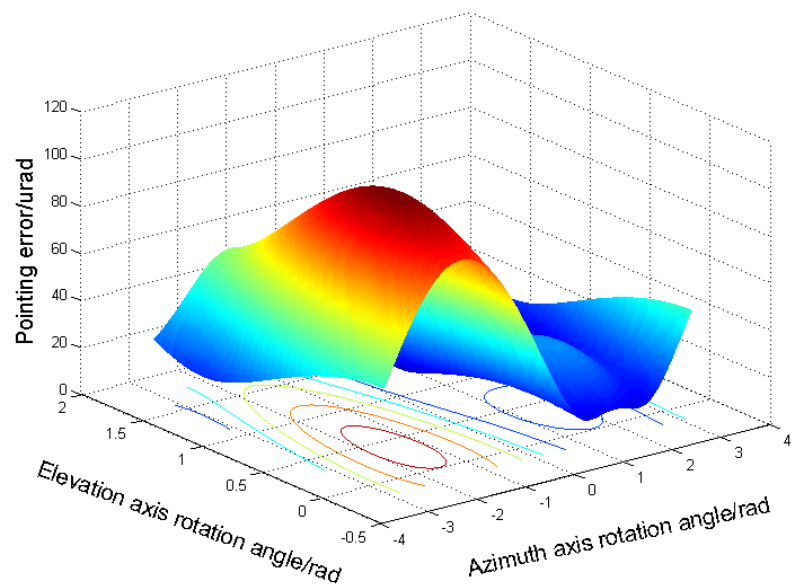

a

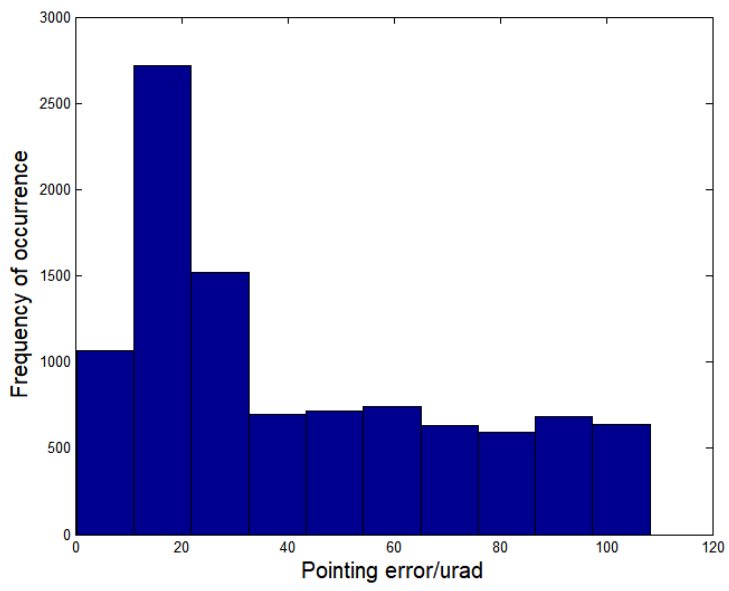

b

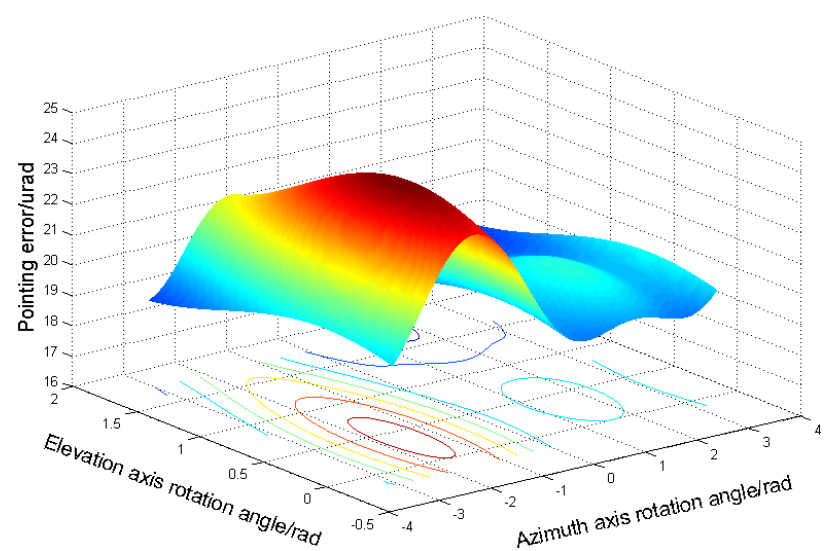

Fig. 10. The COE of signal path distribution with axis rotate angle of (a) $\delta_{1}=2^{\prime \prime}, \delta_{2}=5^{\prime \prime}$, and (b) error frequency histogram; (c) $\delta_{1}=2^{\prime \prime}, \delta_{2}=-5^{\prime \prime}$, and (d) error frequency histogram. 


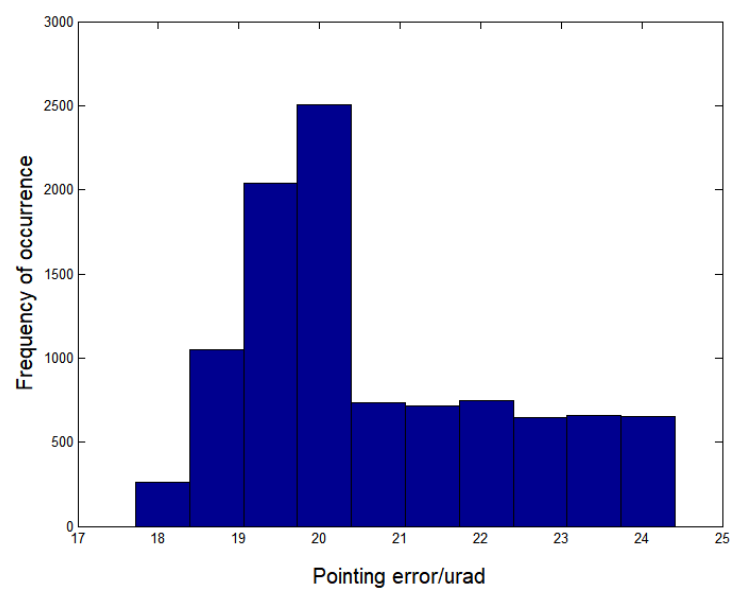

d

Fig. 10. Continued.

the signal path varied with azimuth axis rotate angle significantly, and the error magnitude reduced if the sum of axis errors and reflector errors decreased, and the maximum value of COE reduced from 103 to $24 \mu \mathrm{rad}$ but the position has not changed; it is likely to happen when $\theta_{\mathrm{az}}=-90^{\circ}$.

\section{Coaxiality correction and test experiment}

The coaxiality experimental system is constructed as shown in Fig. 11. The main components of the system are: attenuation link which is used to simulate the real link from 3000 to $5000 \mathrm{~km}$, collimator (which is used to provide the incoming beam, whose focal length is $3 \mathrm{~m}$ ), CCR (the caliber is $30 \mathrm{~mm}$ and the dihedral angle error is $2^{\prime \prime}$ ), the terminal (APT rotate angle range: from $\theta_{\mathrm{az}}=-180^{\circ}$ to $\theta_{\mathrm{az}}=180^{\circ}$ and from $\theta_{\mathrm{el}}=-10^{\circ}$ to $\theta_{\mathrm{el}}=110^{\circ}$ ), the inrun (the slope angle is $45^{\circ}$ ) and the turntable which can rotate 360 degree. The APT is fixed on the slope table and the table is fixed on the turntable, in this way one-dimensional motion can be converted to two-dimensional motion.

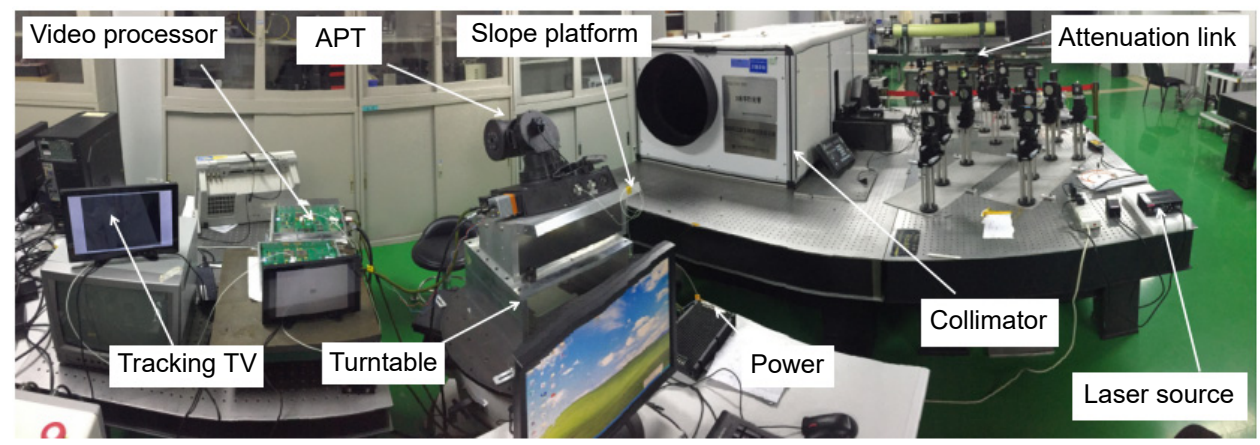

Fig. 11. The coaxiality test experimental system 
When the turntable rotates, the APT rotates in the opposite direction to track the target and will be stopped when it reaches the specified angle. Then the receiving path laser spot location on the detector (both of CTD and FTD) can be recorded. After that, moving the CCR into the antenna face, the outgoing laser will be reflected into the APT system. Then there will be other two laser spots on CTD and FTD, respectively. According to the center of the two spots [13], we can work out the COE. If the COE exceeds the threshold value, it ought to be corrected by adjusting the axis until the COE downgrades within the required threshold. It is important to point out that the dihedral error angle of the CCR will cause wavefront aberration on the retro-diffraction beam. Then the spot energy will become asymmetrical and this will affect the calculation accuracy of COE.

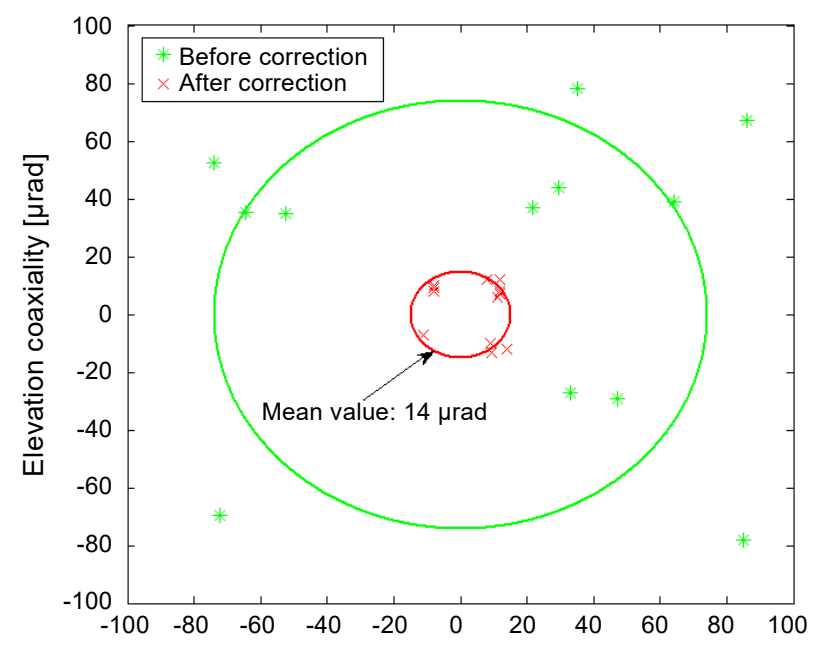

a

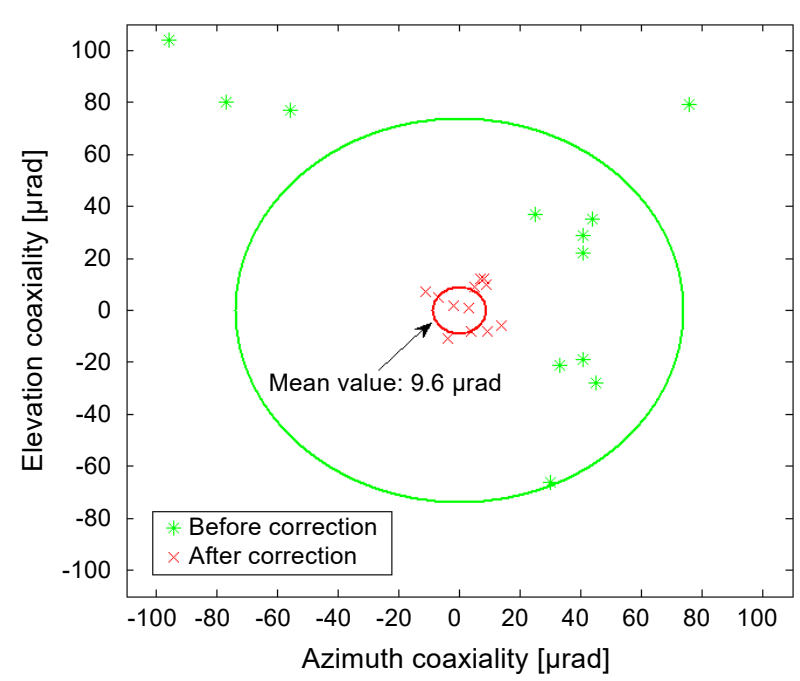

b

Fig. 12. The COE before and after correction of (a) beacon path and (b) signal path. 
For Fresnel diffraction, if the dihedral error angle of the CCR is symmetrically distributed and the value is less than $0.2^{\prime}$, there will also exist energy aberration in the laser spot but its distribution is symmetrical, so the calculation accuracy will not be affected greatly [14].

The COE of both the beacon path and the signal path before and after correction are shown in Fig. 12. The mean value of COE of the beacon path reduced from 70 to $14 \mu \mathrm{rad}$ and the mean value of COE of the signal path reduced from 62 to $9.6 \mu \mathrm{rad}$, which is smaller than the half of communication beam width (which is $30 \mu \mathrm{rad}$ in the experiment), which means that the target will not be lost during tracking.

The COE cannot be corrected limitless, there still exist residual errors. Additionally, there still are some other errors that can contribute to the pointing error such as vibration or point ahead calculation error. Therefore, the COE must be corrected further after satellite is launched. In practice, the closed-loop control method is always used to give the feedback of the COE, then the system can compensate the error according to the feedback, in that way the COE will be further reduced.

\section{Conclusions}

To sum up, high precision beam pointing is the vital indictor of APT system in space laser communication. The pointing error of each optical path and the coaxiality of the APT will be affected by the axis assembly error and the reflector assembly error. In this study, the calculation model that the influence of the assembling error on PE and COE has been established, the quantitative analysis has been performed. The simulation results show that the distribution and magnitude of PE are affected by the sum of the axis assembly error and the reflector assembly error, but the magnitude of coaxiality is just affected by the reflector assembly error. Furthermore, the PE easily becomes bigger at the axis aiming the dead zone such as $\theta_{\mathrm{az}}=-180^{\circ}, 0^{\circ}, 180^{\circ}$ or $\theta_{\mathrm{el}}=+90^{\circ}$, so, we should avoid the APT rotates to the dead zone as far as possible. At last, we corrected and tested the coaxiality. The mean value of the COE of the beacon path and signal path are 14 and $9.6 \mu \mathrm{rad}$, respectively, which is smaller than half beam width. We expect that the research on laser beam pointing error can be used in design and assembling process of the APT and will improve its performance.

Acknowledgement - This article was supported by the 135 project of the Institute of Chinese Academy of Sciences (No. Y655811213). The coaxiality experiments were carried out in the key laboratory of space precision measurement technology, Chinese Academy of Sciences.

\section{References}

[1] Zhang F., Han J., RuAn P., Beam pointing analysis and a novel coarse pointing assembly design in space laser communication, Optik 189, 2019,pp. 130-147, DOI: 10.1016/j.ijleo.2019.05.079.

[2] Ding S., SAN X., Gao S., Ni Y., WANG J., Laser communication pointing errors caused by bending deformation of the altitude axis of a T-shaped altitude-azimuth mount, Applied Optics 58(30), 2019, pp. 8141-8147, DOI: 10.1364/AO.58.008141. 
[3] Jiang H., An Y., Zhang Y., Jiang L., Zhao Y., Dong K., Zhang P., Wang C., Zhan J., Analysis of the status quo, development trend and key technologies of space laser communication, Journal of Spacecraft TT\&C Technology 34(3), 2015, pp. 207-217.

[4] Li H., Han K., Wang X., He S., Wu Q., Xu Z., A compact and lightweight two-dimensional gimbal for inter-satellite laser communication applications, Optics Express 27(17), 2019, pp. 24060-24071, DOI: $10.1364 / \mathrm{OE} .27 .024060$.

[5] Chen G., Xue B., Yang J., Zhao Y., Wang X., He J., Polarization properties of calibration reflector system in the polarization-modulated space laser communication, Optics Communications 430, 2019, pp. 311-317, DOI: 10.1016/j.optcom.2018.06.058.

[6] Zhang L., Wu Z., Closed-form suboptimal maximum-likelihood sequence detection for free-space optical communications, Applied Optics 51(27), 2012, pp. 6441-6447, DOI: 10.1364/AO.51.006441.

[7] Wu Feng, Yu Siyuan, Zhou Jie, Ma Jing, Tan Liying, Analysis of the stability of bidirectional beam tracking in inter-satellite optical communication, Chinese Journal of Lasers 40(11), 2013, article 1105003, DOI: $10.3788 /$ cj1201340.1105003.

[8] Tan L., Song Y., Ma J., Yu S., Han Q., Jiang Y., Wang J., Fu S., Pointing error due to temperature distribution of SiC reflectors in intersatellite laser communications, Applied Optics 49(22), 2010, pp. 4168-4174, DOI: 10.1364/AO.49.004168.

[9] Friederichs L., Sterr U., Dallmann D., Vibration influence on hit probability during beaconless spatial acquisition, Journal of Lightwave Technology 34(10), 2016, pp. 2500-2509, DOI: 10.1109/ JLT.2016.2542918.

[10] Wu S., TAn L., Yu S., MA J., Analysis and correction of axis error in periscope-type optical communication terminals, Optics \& Laser Technology 46, 2013, pp. 127-133, DOI: 10.1016/j.optlastec. 2012.04.035.

[11] Feng D.Y., Gao Y.G., Zhang W.B., Elimination of shafting errors in photoelectrical theodolites with standard-bearings, Optics and Precision Engineering 19, 2011, pp. 605-611.

[12] Qian Feng, Research on the High Precision ATP System in Satellite-to-Earth Quantum Communications, University of Chinese Academy of Sciences, 2014, pp. 87-91.

[13] Wu J., Chen Y., Gao S., Li Y., Wu Z., Improved measurement accuracy of spot position on an In GaAs quadrant detector, Applied Optics 54(27), 2015, pp. 8049-8054, DOI: 10.1364/AO.54.008049.

[14] Sнг Y., Li S., Zhоu H., Weng X.T., MATLAB and its application to solve Fresnel diffraction of the cube corner prism, Optoelectronic Technology \& Information 18(3), 2005, pp. 85-88.

Received March 13, 2020

in revise form April 26, 2020 